[0212-7199 (2004) 21: 12; pp 585-589] ANALES DE MEDICINA INTERNA Copyright (C) 2004 ARAN EDICIONES, S.L

AN. MED. INTERna (Madrid) Vol. 21, N. ${ }^{\circ} 12$, pp. 585-589, 2004

\title{
¿Son los servicios de urgencias hospitalarios un lugar adecuado para morir?
}

\author{
O. RODRÍGUEZ MAROTO, S. LLORENTE ÁLVAREZ'1 M. CASANUEVA GUTIÉRREZ, \\ B. ÁLVAREZ ÁLVAREZ, G. DE LA RIVA MIRANDA
}

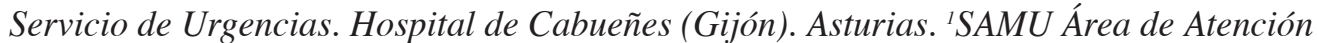
a las Urgencias y Emergencias Médicas. Servicio de Salud del Principado de Asturias

ARE HOSPITAL EMERGENCY SERVICES AN ADEQUATE PLACE TO

DIE?

\section{RESUMEN}

Objetivos: Analizar el proceso asistencial de los fallecidos en un Servicio de Urgencias.

Métodos: Estudio descriptivo retrospectivo de los fallecidos durante el año 2000 analizando las variables clínicas, los tiempos de triage, atención y estancia total, pruebas diagnósticas, tratamientos, así como el lugar de fallecimiento dentro del servicio y la presencia familiar.

Resultados: Se incluyeron 146 pacientes. 80,6\% fueron catalogados como urgencia vital (código rojo), condicionando tiempos de primera exploración significativamente menores que el resto. Al $87 \%$ se le realiza algún tipo de prueba diagnóstica. $40 \%$ recibe maniobras de resucitación y 75,6\% algún tipo de medicación. La analgesia mayor y la sedación fue más empleada en enfermos con patología tumoral. 42,4\% fallecen antes de la primera hora de su ingreso y $83 \%$ antes de seis horas. Los que mueren después de este tiempo lo hacen en el box de observación. $31 \%$ fallece acompañado por algún familiar.

Conclusiones: Nuestro sistema actual de "triage" no parece ser el adecuado para la valoración inicial de los pacientes que fallecen en urgencias. Los que fallecen después de seis horas de su ingreso lo hacen prácticamente en su totalidad en el box de observación, cumpliendo una de las expectativas de funcionamiento del mismo. Observamos una escasa utilización de tratamientos paliativos. La mayoría de nuestros pacientes mueren solos.

PALABRAS CLAVE: Urgencias hospitalarias. Mortalidad. Triage. Tiempos. Tratamientos paliativos.

\begin{abstract}
Objectives: To analyse the assistance procedure to the deceased in a Hospital Emergency Service.

Methods: Descriptive retrospective study of the deceased during 2000 analysing the clinic variables, triage times, attention and total staying, diagnostic tests, treatments as well as the place of decease within the service and the family presence.

Results: 146 patients were included, $80.6 \%$ of which were catalogued as life urgency (red code), conditioning significantly lower first exploration times than the rest. Diagnose tests were made to the $87 \%$, $40 \%$ received resuscitation manoeuvres; finally $75.5 \%$ were given some medication. Major analgesia and sedative treatments were mostly employed with cancer patients. $42.2 \%$ die within the first hour and $83 \%$ before six hours. Those who die after this time die in the observation box. $31 \%$ die accompanied by familiars.

Conclusions: Our present "triage" system does not seem to be the adequate to initially evaluate the patients who die at the Emergency Service. Nearly all who die six hours after their entry die in the observation box, accomplishing one of its operation expectatives. We observe a scarce use of palliative treatments. Most of our patients die alone.
\end{abstract}

KEY WORDS: Emergency services. Mortality. Epidemiology. Palliative treatments.

Rodríguez Maroto O, Llorente Álvarez S, Casanueva Gutiérrez M, Álvarez Álvarez B, de la Riva Miranda G. ¿Son los servicios de urgencias hospitalarios un lugar adecuado para morir? An Med Interna (Madrid) 2004; 21: 585-589.

\section{INTRODUCCIÓN}

La sociedad moderna ha cambiado la orientación de la vida y la muerte hacia una perspectiva dominada por los valores científicos. El proceso de morir ha sufrido cambios desplazándose progresivamente desde el hogar y la familia hacia el médico y los centros hospitalarios (1). Sin embargo los hospitales modernos, con todo su dispositivo técnico, están orientados hacia un objetivo principal que es el restablecimiento de la salud, y ante un paciente terminal, o se cae en la tentación de prolongar la vida por todos los medios posibles, o prevalece un sentimiento de fracaso ("no hay nada más que hacer") (2).

Parece por tanto registrarse una cierta tendencia a acudir a fallecer en el hospital, generando exploraciones y pruebas diagnósticas muchas veces innecesarias, que fortalecen la creencia popular de que siempre se puede "hacer algo más" y condicionando que estos pacientes mueran solos en un medio extraño y sin el apoyo de sus familias. 
La atención al enfermo terminal genera además problemas éticos a la hora de tomar decisiones que a menudo deben ser discutidas con los familiares o responsables legales para hacer una justa utilización de los recursos sanitarios.

\section{MATERIAL Y MÉTODOS}

Estudio descriptivo retrospectivo del total de pacientes que fallecieron en el Servicio de Urgencias del Hospital de Cabueñes durante el año 2000.

El Hospital de Cabueñes es el hospital de cabecera del Área Sanitaria V del Principado de Asturias y atiende a una población de 286.369 habitantes. Cuenta con el apoyo del Hospital de Cruz Roja para el ingreso de ciertas patologías crónicas descompensadas no subsidiarias de nuevos estudios diagnósticos pero que precisan tratamiento o apoyo social.

Durante el año 2000 fueron atendidas en el S. de Urgencias, un total de 81.035 urgencias, de las cuales 47.670 corresponden al área de Urgencias Generales que incluye las secciones de Urgencias ambulatorias, especialidades y urgencias generales como tales, siendo el resto de Pediatría y Traumatología.

El trabajo se realizó en el Servicio de Urgencias, destinado a la atención de los problemas urgentes médico-quirúrgicos durante las 24 horas del día.

$\mathrm{Su}$ estructura funcional es la siguiente: Area de Clasificaciòn y Triage:. Situada a la entrada del SUH, próxima al Servicio de admisión. Aquí los pacientes son recibidos por personal de enfermería, que en función de unos protocolos elaborados y consensuados por el servicio clasifica a los pacientes según su gravedad y el tipo de patología que presentan. Dependiendo de su gravedad se les asigna un código de color que de mayor a menor gravedad será: rojo, amarillo, o verde. Àrea de Urgencias Ambulantes, donde son valorados los pacientes con patología leve, no urgente, cuya atención puede demorarse (código verde). Área de Urgencias Generales (Boxes de Exploración): consta de once huecos (boxes) para pacientes que precisen estar encamados por su situación clínica ò funcional, medicaciones parenterales, oxigenoterapia ò monitorización. En este área son atendidos los pacientes clasificados como códigos "rojos" (patología urgente grave que precisa atención inmediata pues existe riesgo para la vida) y código "amarillo" es decir, aquellos que presentan patología urgente no grave pero cuya atención no puede ser demorada por su patología incapacitante, dolorosa o de necesidad de diagnóstico, pero sin riesgo vital inmediato. Sala de pacientes críticos (paradas). Àrea de Observación (boxes de observación): consta de quince camas para aquellos pacientes que precisen tratamiento u observación por un tiempo presumiblemente inferior a 24 horas, incluyendo aquí también a los que están pendientes de traslado a otro hospital o de ingreso en planta. Es utilizado también cuando la muerte parece inminente e inevitable. Para estos pacientes siempre que es posible se utiliza el box $n^{\circ} 15$ (BOX 15), dado que es una habitación independiente cerrada (no separada por cortinas como el resto de boxes) que permite el acompañamiento permanente de familiares para que si así lo desean, el paciente no permanezca solo.

Se revisaron las historias de todos los pacientes que fallecieron en nuestro servicio durante el año 2000. Mediante una hoja de recogida de datos se registraron las siguientes variables:
Edad, hora que acude: hora de entrada en el servicio de admisión de urgencias; hora "triage": hora en la que el enfermo es clasificado por la enfermera del área de clasificación según su gravedad; hora primera exploración: primera hora registrada en la historia clínica por un médico; hora del fallecimiento. Código de "triage": rojo, amarillo, verde. No consta. Lugar de atención: Urgencias generales, Urgencias traumatológicas, Urgencias pediátricas. Maniobras de Reanimación Cardiopulmonar Avanzada (RCPA) (si/no). Las maniobras de RCP así como los criterios de " no RCP" se realizaron conforme a las recomendaciones del European Resuscitation Council (ERC) (5). Procedimientos diagnósticos: canalización de vena, analítica (alguna de las siguientes: bioquímica sanguínea, hemograma, bioquímica y sedimento de orina, pruebas de coagulación). Radiología simple, electrocardiograma. Otras pruebas de imagen: ecografía, TAC, sonda urinaria. Lugar donde ocurre la muerte: sala de paradas, box de exploración, box de observación, box 15, llega fallecido. Familia presente acompañando al paciente en el momento del fallecimiento. Causa básica de muerte: según la Clasificación Internacional de Enfermedades, $9^{\mathrm{a}}$ edición, Modificación Clínica (CIE-9-MC) .Se consideró como causa de muerte la demencia cuando el paciente presentaba calidad de vida muy mala previamente con Karnofsky menor de 40 y no existía otro motivo claramente definido como causa de la misma. Las neumonías se consideraron dentro del grupo de enfermedades respiratorias y los ACV dentro del grupo de enfermedades del aparato circulatorio. Enfermedades crónicas previas: (sí/no) recogiendo enfermedades previas tipo: neoplasia diseminada, demencia con vida dependiente para actividades de la vida diaria (Karnofsky $<0=50$ ), cardiovasculares, neumológicas, neurológicas, endocrino-metabólicas, digestivas, síndrome de inmunodeficiencia adquirida (SIDA); considerando crónicas aquellas que precisaban tratamiento de forma continuada o producían deterioro de su vida diaria. Tratamiento recibido en Urgencias: analgesia mayor (medicación con opiáceos), sedación (benzodiacepinas, neurolépticos o hipnóticos, con el fin de disminuir la ansiedad, angustia o agitación), otras. Calidad de vida previa según la escala de Karnofsky que puntúa de 10 a 100 de menor a mayor calidad de vida. Fallecimiento previsible a su llegada al Servicio de Urgencias según el paciente presentara a su ingreso en el servicio (4): enfermedad neoplásica avanzada, bradicardia $<$ de 50 lat / min o taquicardia $>$ de 150 lat/min, tensión arterial sistólica < de $80 \mathrm{~mm} \mathrm{Hg}$, bradipnea: menos de 10 respiraciones/min o taquipnea: más de 30 respiraciones/minuto, disminución de nivel de conciencia según escala de Glasgow menor de 10, y además presentaban enfermedad neoplásica avanzada, demencia, neumopatía, cardiopatía, infección por el virus de la inmunodeficiencia humana o cualquier otro proceso crónico con calidad de vida mala o muy mala (índice de Karnofsky igual o inferior a 50) (5).

\section{CÁLCULO DE TIEMPOS DE ESPERA}

- Tiempo triage: minutos transcurridos entre la hora acude y la hora de triage.

- Tiempo de primera exploración: minutos transcurridos entre la hora a la que acude y la hora de primera exploración.

- Tiempo total de estancia: minutos transcurridos entre la hora a la que acude el paciente y la hora del fallecimiento. 
La recogida de datos se realizó en fichas individuales, introduciéndolos en una base de datos y procesándolos con el programa estadístico SPSS-10 (Stadistical Pakage for the Social Sciences).

Las variables cualitativas se expresaron en porcentajes y se compararon utilizando el test de la $\chi^{2}$. Las variables cuantitativas se expresaron como media y se compararon mediante el test paramètrico $\mathrm{T}$ de Student. Para comparar diferencias entre tres o más medias fue utilizado el test de ANOVA con la prueba de Bonferroni para detectar entre que grupos existen diferencias.

\section{RESULTADOS}

En el año 2000, fueron atendidos en el Servicio de Urgencias del Hospital de Cabueñes 81.035 pacientes.

Fueron registrados 149 exitus, todos ellos pertenecientes al área de Urgencias Generales ( 0 exitus en Pediatría, 0 exitus en traumatología.

Fueron analizados 146 pacientes que cumplían los requisitos necesarios de este estudio.

35 pacientes ingresaron en situación de muerte clínica, es decir, no estaba indicada ninguna maniobra de resucitación cuando llegaron a nuestro servicio.

La edad media de los fallecidos fue de 74,78 \pm 13,82 años con rango 18-95.

El grupo diagnóstico de causa de muerte más frecuente según la CIE 9-MC, corresponde al VII (enfermedades del aparato circulatorio) con un 30,1\%, seguido del II (tumores) con un $19,9 \%$ y del VIII (enfermedades del aparato respiratorio) con un $13 \%$.

Utilizando la escala de valoración de calidad de vida de Karnofsky, los pacientes que fallecen en urgencias presentaban un Karnofsky medio de 50,53 con s de 20,35. Un 54,8\% presentaban un karnofsky menor o igual a 50.

Tras aplicar los criterios de muerte predecible explicados en el apartado de pacientes y métodos, encontramos que tras excluir los que ingresan cadáver, en el $97,7 \%$ de los pacientes la muerte era esperada cuando acuden a urgencias.

A su llegada a Urgencias fueron clasificados como patología urgente grave y/o urgencia vital (código rojo) 88 pacientes $(80,6 \%)$, con un tiempo medio de triage de 2,25 minutos (s de $2,74$ y rango $0-13)$.

Un 18,7\% fueron codificados como amarillo y en dos pacientes no consta código de color.

En el $97,7 \%$ de los pacientes la muerte era esperada cuando acuden a urgencias.

Excluidos aquellos que ingresaron cadáver en el servicio (35), se hace venoclisis a 96 pacientes, $(87,38 \%)$. Las pruebas diagnósticas que se realizaron se muestran en la tabla I.

De los 111 pacientes que fallecen dentro del Servicio, el $39,6 \%$ recibieron algún tipo de soporte vital avanzado.

Los pacientes con calidad de vida previa aceptable (Karnofsky > 50) recibieron maniobras de RCP con más frecuencia que los que presentaban muy mala calidad de vida $(72,72 \%$ frente a $21,15 \%) \mathrm{p}<0,001$.

La edad media fue significativamente menor para los pacientes subsidiarios de maniobras de RCP avanzada, $(\mathrm{p}<0,05)$ (Tabla II)

Recibieron algún tipo de medicación 84 pacientes $(75,6 \%)$, siendo esta analgesia mayor en 29 y sedación en 15 .
TABLA I

\begin{tabular}{lcc}
\multicolumn{3}{c}{ PROCEDIMIENTOS DIAGNÓSTICOS } \\
\hline Nnalítica & $N$ & $\%$ \\
EKG & 72 & 64,86 \\
Radiología & 55 & 49,54 \\
Ecografía & 50 & 45,04 \\
TAC & 2 & 1,80 \\
Sonda urinaria & 9 & 8,10 \\
\hline
\end{tabular}

Como se ve en la figura 1, tanto la analgesia mayor como la sedación, fue más indicada en los pacientes tumorales que en los no tumorales $\mathrm{p}<0,05$.

En la tabla III, se expresan los tiempos medios en minutos de: triage, primera exploración y estancia total con sus desviaciones estándar. Al no recibir asistencia, hemos excluido los 35 pacientes que ingresaron cadáver en el estudio del segundo y tercer tiempo. Los tiempos medios de primera exploración para los pacientes codificados a su ingreso como rojos fueron de 13,36 minutos $\pm 33,54$ (rango 1-274) significativamente menores que para los que fueron clasificados como amarillos con media de 66,28 minutos $\pm 74,55$ (rango 1-324) ( $p<0,05)$.

Los tiempos medios de estancia total según el lugar del servicio donde fallecen estos pacientes son los que muestra la tabla IV.

Un $40,5 \%$ de los pacientes, tuvieron un tiempo de estancia total menor o igual a 1 hora, un 42,34\% un tiempo entre 1 y 6 horas y un 17,11\% más de 6 horas. En la figura 2, se ve la distribución de estas franjas horarias según el lugar de fallecimiento, observando que los tiempos de estancia de más de 6 horas corresponden prácticamente en su totalidad (excepto un

TABLA II

EDAD DE LOS FALLECIDOS SEGÚN HALLAN RECIBIDO MANIOBRAS DE RCP

\begin{tabular}{lccccc}
\hline RCP & $N$ & Edad media & $D E$ & Max. & Min. \\
\hline Si & 44 & 68,79 & 14,30 & 90 & 24 \\
No & 102 & 77,36 & 12,84 & 95 & 18 \\
Total & 146 & & & & \\
\hline
\end{tabular}

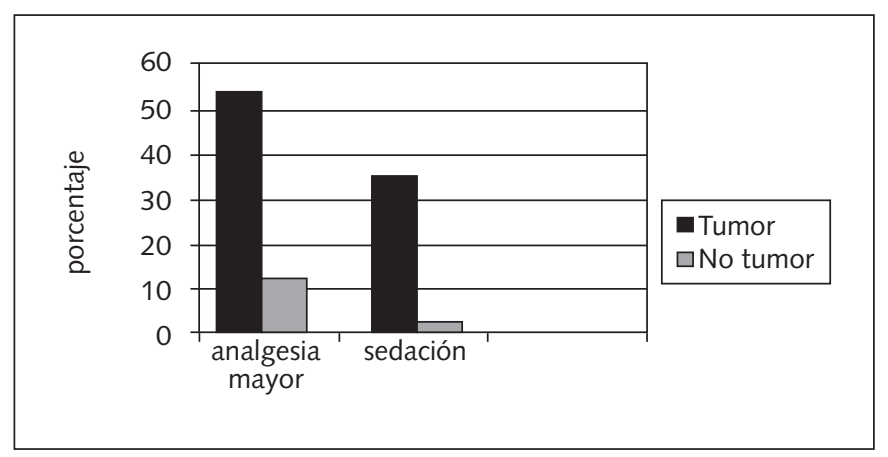

Fig. 1. Tratamiento con analgesia y sedación según patología. 
TABLA III

TIEMPOS DEL PROCESO ASISTENCIAL

\begin{tabular}{lccccc}
\hline$T^{\circ}$ en minutos & Media & $D E$ & Min. & Max. & $N$ \\
\hline$T^{\circ}$ triage & 2,24 & 2,73 & 1 & 13 & 146 \\
$T^{\circ} 1^{\circ}$ exploraciòn & 23,12 & 48,38 & 1 & 324 & 111 \\
$T^{\circ}$ estancia & 235,61 & 352,13 & 5 & 1.834 & 111 \\
\hline
\end{tabular}

TABLA IV

TIEMPOS DE ESTANCIA TOTAL, SEGÚN LUGAR DE FALLECIMIENTO

\begin{tabular}{lcccrc}
\hline$T^{\circ}$ en minutos & Media & $D E$ & Min. & Max. & $N$ \\
\hline Sala RCP & 56,33 & 65,44 & 356 & 8 & 42 \\
Box. urgencias & 128,65 & 104,5 & 406 & 5 & 42 \\
Box. observaciòn & 568,35 & 303,96 & 968 & 224 & 9 \\
Box. 15 & 755,55 & 514,82 & 1834 & 77 & 18 \\
\hline
\end{tabular}

paciente) a las muertes que ocurren en el box de observación y en especial en el box 15.

En la tabla $\mathrm{V}$ y en la figura 3 puede verse como el tiempo total de estancia medio, fue significativamente mayor para los enfermos que fallecieron por causa tumoral que por cualquiera de los otros dos grupos diagnósticos $(\mathrm{p}<0,05)$.

Un $30,8 \%$ de los pacientes fallecen acompañados por algún familiar y el lugar de èxitus dentro el servicio es el que indica la figura 4.

\section{DISCUSIÓN}

A pesar de que prácticamente la totalidad de los pacientes estudiados presentaban a su ingreso en el servicio signos clínicos que hacían prever un desenlace fatal, solo el 80,6\% fue codificado como urgencia vital (código rojo). Coincidiendo con el estudio sobre tiempos de demora en nuestro servicio de urgencias de la Dra. Álvarez (6), los tiempos de primera exploración (minutos que tardaron en ser valorados por el médico desde su llegada al hospital) fueron significativamente menores para los pacientes codificados con color rojo que para los amarillos. Esto condicionó que estos últimos vieran demorada su atención en una media de 60 minutos.

Aunque el alcance del estudio no permite demostrar la causa de esta posible distorsión en el triage pensamos que

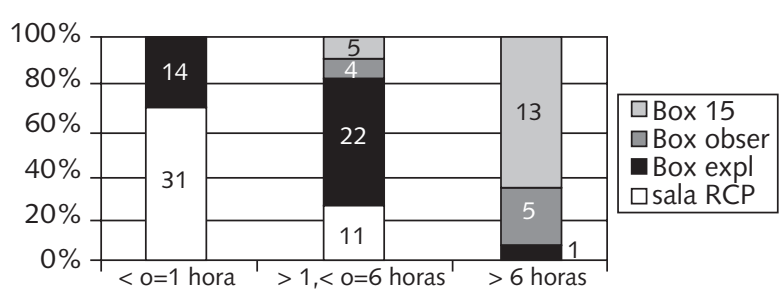

Fig. 2. Tiempo de estancia total según lugar de muerte.

\begin{tabular}{lccccc}
\multicolumn{6}{c}{ TABLA V } \\
TIEMPO DE ESTANCIA TOTAL SEGÚN GRUPOS \\
DIAGNÓSTICOS \\
\hline$T^{\circ}$ en minutos & Media & DE & Min. & Max. & $N$ \\
\hline Tumor & 326,037 & 348,25 & 6 & 1453 & 27 \\
Cardiovascular & 191,69 & 367,52 & 5 & 1834 & 32 \\
Respiratorio & 169,64 & 238,09 & 13 & 909 & 14 \\
\hline
\end{tabular}

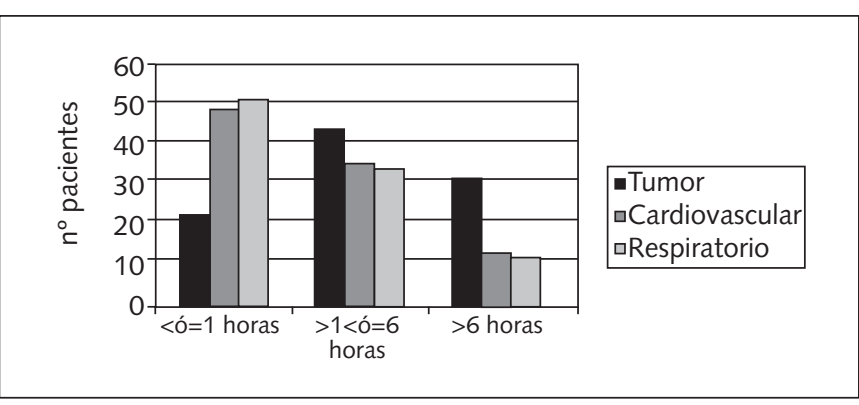

Fig. 3. Tiempo de estancias por grupos diagnósticos.

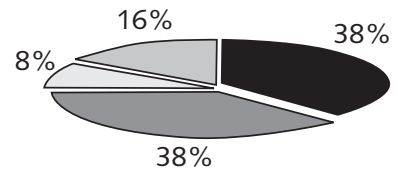

Sala RCP Box exploración $\square$ Box observación $\square \operatorname{Box} 15$

Fig. 4. Lugar de fallecimiento.

además de la variabilidad individual del personal que valora al paciente y las características de la patología aguda que en el momento de ingreso presentaban, posiblemente existen deficiencias en los protocolos de clasificación para valorar la urgencia y atención de los pacientes con mala calidad de vida y enfermedades terminales. La definición de los distintos códigos de triage (7) deja una laguna para la clasificación de aquellos pacientes con enfermedades terminales y degenerativas con mala calidad de vida, en los que aunque parece existir un riesgo inmediato para la vida, no pueden ser considerados como emergencias, pero sí precisarían una atención rápida para garantizarles un mayor confort médico y humano.

Más de la mitad de los fallecidos presentaban previamente calidad de vida muy mala con incapacidad severa y necesidad de asistencia y cuidados sanitarios frecuentes.

La realización de pruebas diagnósticas a estos pacientes es muy inferior a la realizada habitualmente en el área de Urgencias Generales de nuestro servicio, donde prácticamente se hace algún tipo de prueba diagnóstica al $100 \%$ de los pacientes (8). La mayoría de los fallecidos fueron codificados como urgencias vitales (código rojo) y cabría esperar un mayor porcentaje de procedimientos. La explicación a este fenómeno podría deberse a que como vimos en la figura 10 , más de un 
tercio de nuestros pacientes estudio fallecieron antes de una hora de su ingreso en el servicio, con calidad de vida previa mala y criterios de fallecimiento inminente.

Un 39,6\% recibieron maniobras de Reanimación Cardiopulmonar y estas fueron más frecuentes en los pacientes más jóvenes y con mejor calidad de vida. Aunque este estudio no profundizó sobre la idoneidad de estas maniobras, vemos que el mayor número de estas maniobras se hicieron en los pacientes que fallecieron en menos de una hora desde su ingreso en el servicio. El desconocimiento frecuentemente de la historia clínica del paciente y de su calidad de vida previa en los primeros minutos del ingreso hace que se inicien en ocasiones maniobras de resucitación que pudieran no estar indicadas.

En cuanto a los tratamientos administrados en urgencias quisimos detenernos en el uso de medicación paliativa dadas las características de enfermedad terminal y muerte inevitable que presentaban gran parte de nuestros pacientes. Vemos que en muy pocos fue indicada analgesia mayor y sedación en su proceso de muerte .

Son los pacientes tumorales en los que hacemos más uso de la analgesia y sedación, lo que quizá se deba a una mejor formación en el cuidado de estos pacientes, o a una mayor sensibilización con el sufrimiento del enfermo terminal y su familia. Posiblemente los facultativos de urgencias tendemos a adoptar una actitud más paliativa si contamos con información clínica fiable sobre la existencia de una enfermedad terminal.

Muchos son los estudios recogidos en la literatura médica sobre el uso de opioides en el tratamiento sintomático de la enfermedad neoplásica terminal e incluso también de otros dolores crónicos (9-11), pero es más difícil encontrar protocolos sobre ayuda a la muerte de otras patologías. Quizá bajo esta actitud puedan prevalecer antiguos prejuicios que hacen confundir la ayuda a una muerte digna con la eutanasia.

Una cuarta parte de los pacientes llegaron en muerte clínica y de los que acceden con vida al mismo un $75 \%$ fallecen en la sala de críticos o en los boxes de exploración durante el proceso asistencial en las primeras 6 horas desde su ingreso en el servicio. El $25 \%$ restante fallece en el box de observación (incluido el box 15), presentando estos, tiempos de estancia significativamente mayores que los anteriores, como corresponde al cumplimiento de una de las expectativas de funcionamiento del box de observación como lugar de espera de la muerte con la progresiva implantación en nuestro servicio de decisiones consensuadas entre el médico y el paciente y sus familiares sobre la necesidad de no medidas agresivas, es decir, conducta expectante ante determinadas situaciones (12).

Destacar que aunque la muerte parece esperada desde el momento del ingreso en la mayoría de los casos y en general son pacientes mayores con calidad de vida muy deteriorada, muy pocos mueren acompañados por sus familiares. Esto podría deberse a diferentes factores: sólo un habitáculo del servicio (box 15) permite el acompañamiento familiar, la muerte sobreviene en gran número de ellos dentro del proceso asistencial( seis primeras horas desde su ingreso) y en este por las características estructurales del servicio, la saturación habitual y nuestra forma de trabajo, no suele permitirse el acompañamiento familiar. Este dato hace reflexionar sobre la necesidad de establecer protocolos de actuación ante estas situaciones que garanticen un mayor confort clínico y humano.

\section{Bibliografía}

1. Sanz Ortiz J. La sociedad, la medicina y la muerte. Med Clin (Barc) 1990; 95: 419-20.

2. Sanz Ortiz J. Principios y práctica de los cuidados paliativos. Med Clin (Barc) 1989; 92: 143-5.

3. Kloeck W, Cummins R, Chamberlain D, Bossaert L, Callanan V, Carli P. The Universal ALS Alorithm: An advisory Stament by the Advanced Life Support Working. Group of the International Liaison Commitee on Resuscitation. Resuscitation 1997; 34: 101-8.

4. Córdoba Victoria A, Delgado Lozano LC, Cabrera Vélez R, Kessler P, Perpiña C, Castro C, Rañada M. Estudio de la mortalidad en el Servicio de Urgencias del Hospital 12 de Octubre durante 1989. An Med Interna (Madrid) 1991; 8: 487-90.

5. Nieto Sánchez A, Arranz Gómez F, Lana Soto R, Torres Villaredo P, Rodríguez Carrillo M, Jiménez de Diego L. Análisis descriptivo de la mortalidad en un servicio de urgencias terciario. XII Congreso Nacional de la Sociedad de Medicina de Urgencias y Emergencias. Santander 2000.

6. Alvarez Alvarez B, Gorostidi Pérz J, Rodríguez Maroto O., Antuña Egocheaga A, Alonso Alonso P. Estudio del triage y tiempos de espera en un servicio de urgencias hospitalario. Emergencias 1998; 10: 100-4.

7. Bustamante Vega R, Castro Maderal S, Alonso Blas JC. Priorización de asistencia en Urgencias: triage. Congreso de la Sociedad Española de Medicina de Emergencias. Oviedo, Junio 1995.

8. Llorente Álvarez S. Determinantes de los tiempos de asistencia en un servicio de urgencias hospitalario. Tesis doctoral. Departamento de Medicina Preventiva. Universidad de Oviedo.

9. Eladi Baños J. Dolor y opioides: razones de una sinrazón. Med Clin (Barc) 1997; 109: 294-6.

10. Belderrain Belderrain P, García Busto B, Castañón Quiñones E. Atención sanitaria y comunicación con el enfermo oncológico en situación terminal y su familia en un área de salud. Atención Primaria 1999; 24 285-8.

11. López Martínez de Pinillos E, Pérez de Lucas N, López Martínez de Pinillos R., Martín Serrano P, Sanz Juez F, Alonso Salazar MT. Atención a pacientes oncológicos terminales. Emergencias 1999; 11: 398402.

12. Montero Pérez FJ, Calderón de la Barca Gázquez JM, Jiménez Murillo L, Berlango Jiménez A, Pérz Torres I, Pérula de Torres L. Situación actual de los Servicios de Urgencias Hospitalarios en España (IV): Áreas de Observación. Emergencias 2000; 12: 259-68. 